\title{
Effect of carbamazepine and gabapentin on excitability in the trigeminal subnucleus caudalis of neonatal rats using a voltage-sensitive dye imaging technique
}

Akiko Matsumoto ${ }^{1 \dagger}$, Hirofumi Arisaka ${ }^{1 \dagger}$, Yuki Hosokawa², Shigeki Sakuraba', Takeo Sugita ${ }^{1}$, Nobuo Umezawa', Yuki Kaku ${ }^{3}$, Kazu-ichi Yoshida' and Shun-ichi Kuwana ${ }^{4^{*}}$

\begin{abstract}
Background: The antiepileptic drugs carbamazepine and gabapentin are effective in treating neuropathic pain and trigeminal neuralgia. In the present study, to analyze the effects of carbamazepine and gabapentin on neuronal excitation in the spinal trigeminal subnucleus caudalis $(\mathrm{Sp} 5 \mathrm{c}$ ) in the medulla oblongata, we recorded temporal changes in nociceptive afferent activity in the Sp5c of trigeminal nerve-attached brainstem slices of neonatal rats using a voltage-sensitive dye imaging technique.

Results: Electrical stimulation of the trigeminal nerve rootlet evoked changes in the fluorescence intensity of dye in the Sp5c. The optical signals were composed of two phases, a fast component with a sharp peak followed by a long-lasting component with a period of more than 500 ms. This evoked excitation was not influenced by administration of carbamazepine (10, 100 and 1,000 $\mu \mathrm{M}$ ) or gabapentin (1 and $10 \mu \mathrm{M})$, but was increased by administration of $100 \mu \mathrm{M}$ gabapentin. This evoked excitation was increased further in low $\mathrm{Mg}^{2+}(0.8 \mathrm{mM})$ conditions, and this effect of low $\mathrm{Mg}^{2+}$ concentration was antagonized by $30 \mu \mathrm{M}$ DL-2-amino-5-phosphonopentanoic acid (AP5), a N-methyl-D-aspartate (NMDA) receptor blocker. The increased excitation in low $\mathrm{Mg}^{2+}$ conditions was also antagonized by carbamazepine $(1,000 \mu \mathrm{M})$ and gabapentin $(100 \mu \mathrm{M})$.

Conclusion: Carbamazepine and gabapentin did not decrease electrically evoked excitation in the Sp5c in control conditions. Further excitation in low $\mathrm{Mg}^{2+}$ conditions was antagonized by the NMDA receptor blocker AP5. Carbamazepine and gabapentin had similar effects to AP5 on evoked excitation in the Sp5c in low $\mathrm{Mg}^{2+}$ conditions. Thus, we concluded that carbamazepine and gabapentin may act by blocking NMDA receptors in the Sp $5 c$, which contributes to its anti-hypersensitivity in neuropathic pain.
\end{abstract}

Keywords: Carbamazepine, Gabapentin, Rat brainstem, Spinal trigeminal nucleus, Voltage-sensitive dye imaging

\section{Background}

The antiepileptic agents carbamazepine and gabapentin are effective against neuropathic pain and trigeminal neuralgia $[1,2]$. However, the action of these antiepileptic drugs on neuronal activity in the brain may not be

\footnotetext{
*Correspondence: s-kuwana@uekusa.ac.jp

${ }^{\dagger}$ Akiko Matsumoto and Hirofumi Arisaka equally contributed to this work

${ }^{4}$ Faculty of Health Sciences, Uekusa Gakuen University, Ogura-cho,

Wakaba-ku, Chiba 264-0007, Japan

Full list of author information is available at the end of the article
}

simple. A large body of evidence indicates that carbamazepine may interact with different types of ion channels and synaptic transmission [3, 4]. The molecular targets for ion channels have generally been voltage-gated $\mathrm{Na}^{+}$ channels [5], $\mathrm{Ca}^{2+}$ channels [6] and $\mathrm{K}^{+}$channels [7]. An increasing number of findings indicate that carbamazepine induces the inhibition of glutamate release [8], inhibition of an adenosine receptor [9] and modulation of neuromodulator levels, such as those of serotonin, dopamin and cyclic adenosine monophosphate (AMP) [3]. In 
addition, the effects of gabapentin on neural activity are not explained by a single mechanism $[10,11]$. Although gabapentin is a structural analogue of $\gamma$ amino-butyric acid (GABA), it has no affinity for GABA receptors. The main target of gabapentin is synaptic transmission, where it inhibits voltage-gated $\mathrm{Ca}^{2+}$ channels in the presynaptic membrane, which inhibits the release of glutamate and substance $P$ [12]. Recent evidence suggests that other actions of gabapentin include inhibition of glutamatergic $N$-methyl-D-aspartate (NMDA) receptors [13] and an increase in GABA release [14].

To study the neural mechanisms of trigeminal neuralgia, trigeminal nerve-attached brainstem preparations from neonatal rats are used $[15,16]$. In electrophysiological studies, activity-dependent neuronal hyperexcitability, so-called central sensitization, has been reported in the spinal trigeminal subnucleus caudalis $(\mathrm{Sp} 5 \mathrm{c})$, which receives nociceptive information from the orofacial area. Furthermore, such studies have shown that NMDA receptors contribute substantially to polysynaptic transmission in the $\mathrm{Sp} 5 \mathrm{c}$ and to long-term potentiation.

To analyze the spatial dynamics of neuronal excitation propagation in the $\mathrm{Sp} 5 \mathrm{c}$, it is possible to use optical imaging analysis and voltage-sensitive dyes [17, 18]. A previous study using trigeminal nerve-attached brainstem slices from postnatal rats showed that synaptic transmission via unmyelinated afferents in the Sp5c was mediated substantially by NMDA receptors [19]. In the present study, we examined the effects of carbamazepine and gabapentin on excitability in the Sp5c of neonatal rats using an optical imaging technique. Furthermore, we confirmed the contribution of NMDA receptors to enhanced excitability in the $\mathrm{Sp} 5 \mathrm{c}$ in low $\mathrm{Mg}^{2+}$ concentration conditions.

\section{Results}

Influence of carbamazepine on evoked excitation in the Sp5c

The influence of drug administration on evoked excitation was examined using trigeminal nerve-attached brainstem sagittal slice preparations, as shown in Figure 1. Figure 2 shows the influence of carbamazepine at different concentrations $(10,100$ or $1,000 \mu \mathrm{M})$ on evoked excitation in the Sp5c. During superfusion with mock cerebrospinal fluid (CSF) (Figure 2a), the optical signals were composed of two phases, a fast component with a sharp peak followed by a long-lasting component with a period of more than $500 \mathrm{~ms}$. The time delay from stimulation to the peak of the first component was $36.8 \pm 8.3 \mathrm{~ms}(\mathrm{n}=6)$. The distance from the nerve rootlet to the Sp5c was approximately $4.0 \mathrm{~mm}$. Therefore, the conduction velocity was approximately $0.11 \mathrm{~m} / \mathrm{s}$.
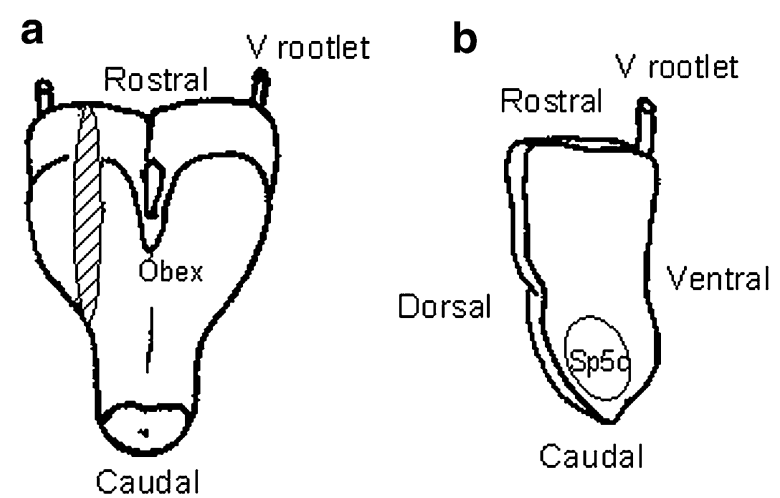

Figure 1 Preparations used for membrane potential imaging. a Isolated trigeminal nerve-brainstem preparation. The dorsal side is shown at the front, and the sagittal sectional surface is shown by hatching. b Trigeminal nerve-attached brainstem sagittal slice preparation. The preparation was placed with the sagittal sectional surface upwards for measurement. Electric stimulation was applied by sucking the trigeminal nerve root with a suction electrode for all preparations. Sp5c spinal trigeminal subnucleus caudalis, V rootlet trigeminal nerve rootlet.

The intensity and time course of the optical signal did not alter significantly after switching to superfusion with 1,000 $\mu \mathrm{M}$ carbamazepine-containing mock CSF (Figure 2b). The left-hand panels in Figure 2a, b show fluorescent signal images at $165 \mathrm{~ms}$ after electrical stimulation during superfusion with mock CSF and with 1,000 $\mu \mathrm{M}$ carbamazepine-containing mock CSF, respectively. The right-hand panels in Figure 2a, b show time-courses of fluorescent signal changes during superfusion with mock CSF (Figure 2a) and with $1,000 \mu \mathrm{M}$ carbamazepine-containing CSF (Figure 2b), respectively. When electrical stimulation was applied in the presence of $1,000 \mu \mathrm{M}$ carbamazepine, the timecourse of Sp5c excitement showed a sharp peak after stimulation followed by a slow decline, similar to that in the control. To analyze signal amplitudes during superfusion with 10,100 and $1,000 \mu \mathrm{M}$ carbamazepine, fluorescence signal amplitude was indicated as the percent amplitude of the control conditions. The peak amplitude of the first component seemed to decrease slightly to $96.0 \pm 12.5 \%$ during superfusion of $1,000 \mu \mathrm{M}$ carbamazepine, but the difference was not significant (Figure 2c). The peak amplitude of the first component was not affected by any concentration of carbamazepine (Figure 2c). To evaluate the effect of carbamazepine on the long-lasting component, we measured the evoked signal at 165 and $385 \mathrm{~ms}$ after stimulation (Figure 2d, e), but the signal amplitude of the long-lasting component was also unaffected by carbamazepine (Figure 2d, e). 


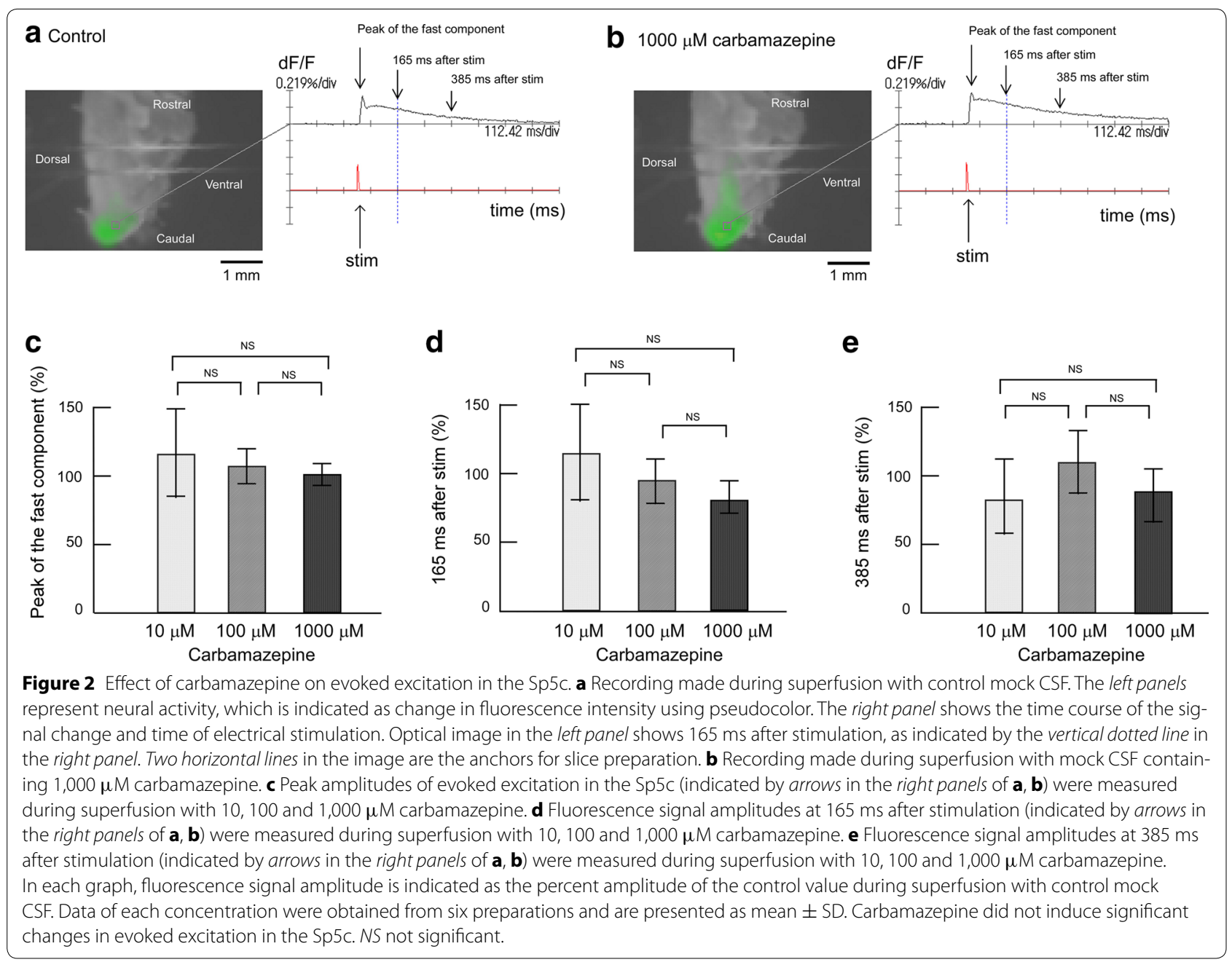

\section{Influence of gabapentin on evoked excitation in the Sp5c}

The dose-response relationship of gabapentin with the evoked excitation was examined using trigeminal nerve-attached brainstem sagittal slice preparations (Figure 3). When electrical stimulation was applied in the presence of 1 or $10 \mu \mathrm{M}$ gabapentin, the timecourse of Sp5c excitement showed a sharp peak after stimulation followed by a slow decline, similar to that in the control conditions. The signal amplitudes of the fast and long-lasting components were not affected by 1 or $10 \mu \mathrm{M}$ gabapentin (Figure $3 \mathrm{c}-\mathrm{e}$ ). Of note, $100 \mu \mathrm{M}$ gabapentin significantly increased the peak amplitude of the fast component to $178 \pm 5.66 \%$ ( $p<0.01$ ). Signal amplitudes at 165 and $385 \mathrm{~ms}$ after stimulation increased to $179 \pm 23.5 \%$ (Figure $3 \mathrm{~d}$ ) and $240 \pm 31.2 \%$, respectively (Figure 3e). Thus, $100 \mu \mathrm{M}$ gabapentin induced further excitation in the electrical trigeminal nerve root stimulation-induced excitement of the Sp5c.

\section{Influence of low $\mathrm{Mg}^{2+}$ concentration and an NMDA} antagonist on evoked excitation in the Sp5c

The influence of low $\mathrm{Mg}^{2+}$ concentration $(0.8 \mathrm{mM})$ on evoked excitation in the Sp5c was observed using trigeminal nerve-attached brainstem sagittal slice preparations (Figure 4). When electrical stimulation was applied during superfusion with low $\mathrm{Mg}^{2+}$ concentration solution, the peak of the fast component after stimulation increased to $177 \pm 30.6 \%$. Furthermore, the signal amplitude at 165 and $385 \mathrm{~ms}$ after electrical stimulation increased markedly over $250 \%$ during superfusion with low $\mathrm{Mg}^{2+}$ concentration solution (Figure 4), suggesting that the low $\mathrm{Mg}^{2+}$ concentration increased electrical trigeminal nerve root stimulation-induced excitement in the Sp5c. The increase in the long-lasting component with low $\mathrm{Mg}^{2+}$ concentration treatment was greater than that of the fast component. A previous study performed by Takuma [19] showed that increased excitation in the $\mathrm{Sp} 5 \mathrm{c}$ with low $\mathrm{Mg}^{2+}$ concentration treatment was 

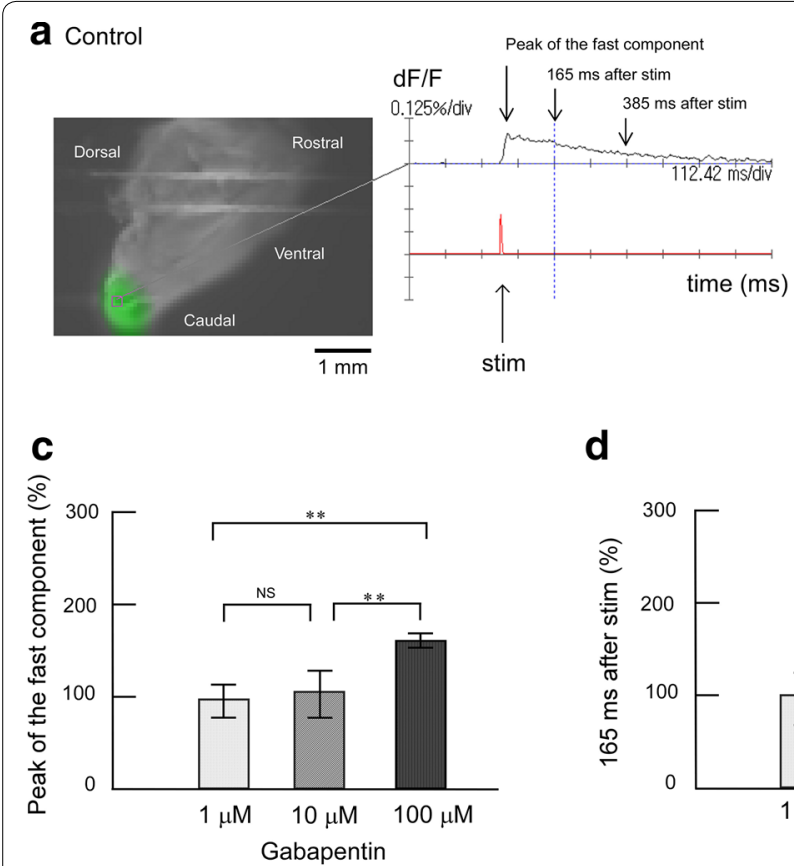
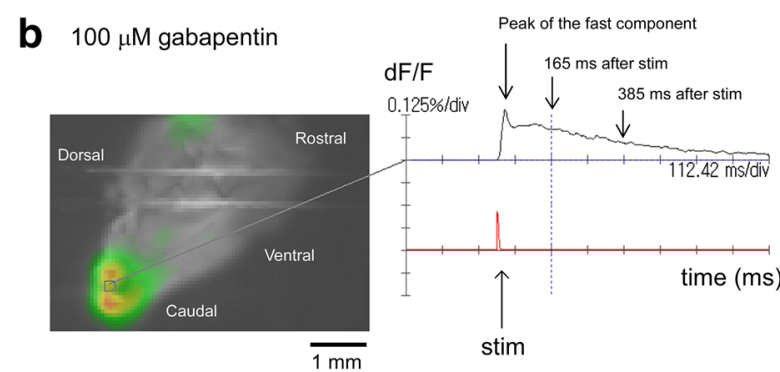

d

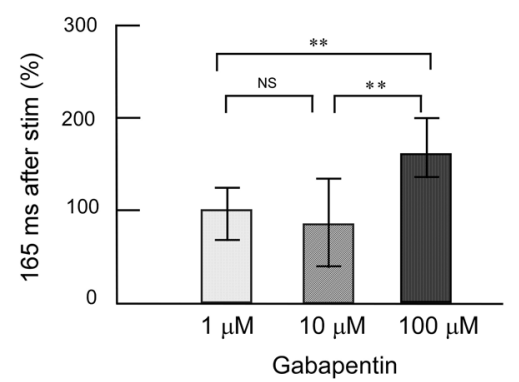

e

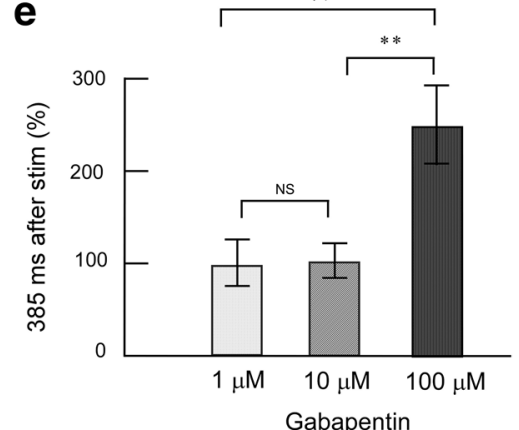

Figure 3 Effect of gabapentin on evoked excitation in the Sp5c. a Recording made during superfusion with control mock CSF. The left panels represent neural activity, which is indicated as changes in fluorescence intensity using pseudocolor. The right panel shows the time course of the signal change and time of electrical stimulation. Optical image in the left panel shows $165 \mathrm{~ms}$ after stimulation, as indicated by the vertical dotted line in the right panel. Two horizontal lines in the image are the anchors for slice preparation. b Recording made during superfusion with mock CSF containing $100 \mu \mathrm{M}$ gabapentin. c Peak amplitudes of evoked excitation in the Sp5c (indicated by arrows in the right panels of $\mathbf{a}$, b) were measured during superfusion with 1, 10 and $100 \mu \mathrm{M}$ gabapentin. $\mathbf{d}$ Fluorescence signal amplitudes at $165 \mathrm{~ms}$ after stimulation (indicated by arrows in the right panels of $\mathbf{a}, \mathbf{b}$ ) were measured during superfusion with 1, 10 and $100 \mu \mathrm{M}$ gabapentin. e Fluorescence signal amplitudes at $385 \mathrm{~ms}$ after stimulation (indicated by arrows in the right panels of $\mathbf{a}, \mathbf{b}$ ) were measured during superfusion with 1, 10 and $100 \mu \mathrm{M}$ gabapentin. In each graph, fluorescence signal amplitude is indicated as the percent amplitude of the control value during superfusion with control mock CSF. Data of each concentration were obtained from six preparations and are presented as mean \pm SD. Administration of 1 and $10 \mu \mathrm{M}$ gabapentin did not induce significant changes in evoked excitation in the Sp5c, but $100 \mu \mathrm{M}$ gabapentin emphasized the evoked excitation. ${ }^{* *} \mathrm{P}<0.01$; NS not significant.

partially antagonized by the NMDA antagonist AP5. We also examined additional superfusion with $30 \mu \mathrm{M}$ AP5 in low $\mathrm{Mg}^{2+}$ conditions. Figure 4c showed that administration of AP5 attenuated the evoked excitement in the $\mathrm{Sp} 5 \mathrm{c}$ and restored it to the control level. The peak amplitude was $126 \pm 19.2 \%$ during superfusion with $30 \mu \mathrm{M}$ AP5 in low $\mathrm{Mg}^{2+}$ conditions (Figure 4d). The amplitude of the long-lasting component was also restored by additional superfusion with $30 \mu \mathrm{M}$ AP5 in low $\mathrm{Mg}^{2+}$ conditions. The amplitudes at 165 and $385 \mathrm{~ms}$ after stimulation decreased to $104 \pm 21.4 \%$ (Figure $4 \mathrm{e}$ ) and $91.4 \pm 31.5 \%$ (Figure 4f), respectively.

\section{Influence of carbamazepine on evoked excitation in the $\mathrm{Sp} 5 \mathrm{C}$ in low $\mathrm{Mg}^{2+}$ conditions}

Figure 5 shows the effect of carbamazepine in low $\mathrm{Mg}^{2+}$ conditions on evoked excitation in the Sp5c. Superfusion with a low $\mathrm{Mg}^{2+}$ concentration solution potentiated the evoked excitation. The effect of these conditions on the long-lasting component $(205 \pm 67.3 \%$ at $165 \mathrm{~ms}$ and $237 \pm 40.8 \%$ at $385 \mathrm{~ms}$ after stimulation) was more marked than that on the fast component (162 $\pm 63.8 \%)$. Additional administration of $1,000 \mu \mathrm{M}$ carbamazepine in low $\mathrm{Mg}^{2+}$ conditions attenuated the evoked excitation to the level of the control conditions (Figure 5). The peak amplitude was $130 \pm 40.2 \%$ during superfusion (Figure 5d). The amplitude of the long-lasting component was also restored by additional superfusion with carbamazepine in low $\mathrm{Mg}^{2+}$ conditions. The amplitudes at 165 and $385 \mathrm{~ms}$ after stimulation decreased to $113 \pm 58.5 \%$ (Figure 5e) and $119 \pm 60.7 \%$ (Figure 5f), respectively. The effect of carbamazepine in low $\mathrm{Mg}^{2+}$ conditions was similar to that of AP5.

\section{Influence of gabapentin on evoked excitation in the Sp5c in low $\mathrm{Mg}^{2+}$ conditions}

Figure 6 shows the effect of gabapentin on evoked excitation in the Sp5c in low $\mathrm{Mg}^{2+}$ conditions. Superfusion with low $\mathrm{Mg}^{2+}$ concentration solution caused potentiation of the evoked excitation. The effect of low $\mathrm{Mg}^{2+}$ concentration solution on the long-lasting component $(148 \pm 14.5 \%$ at $165 \mathrm{~ms}$ and $186 \pm 21.5 \%$ 


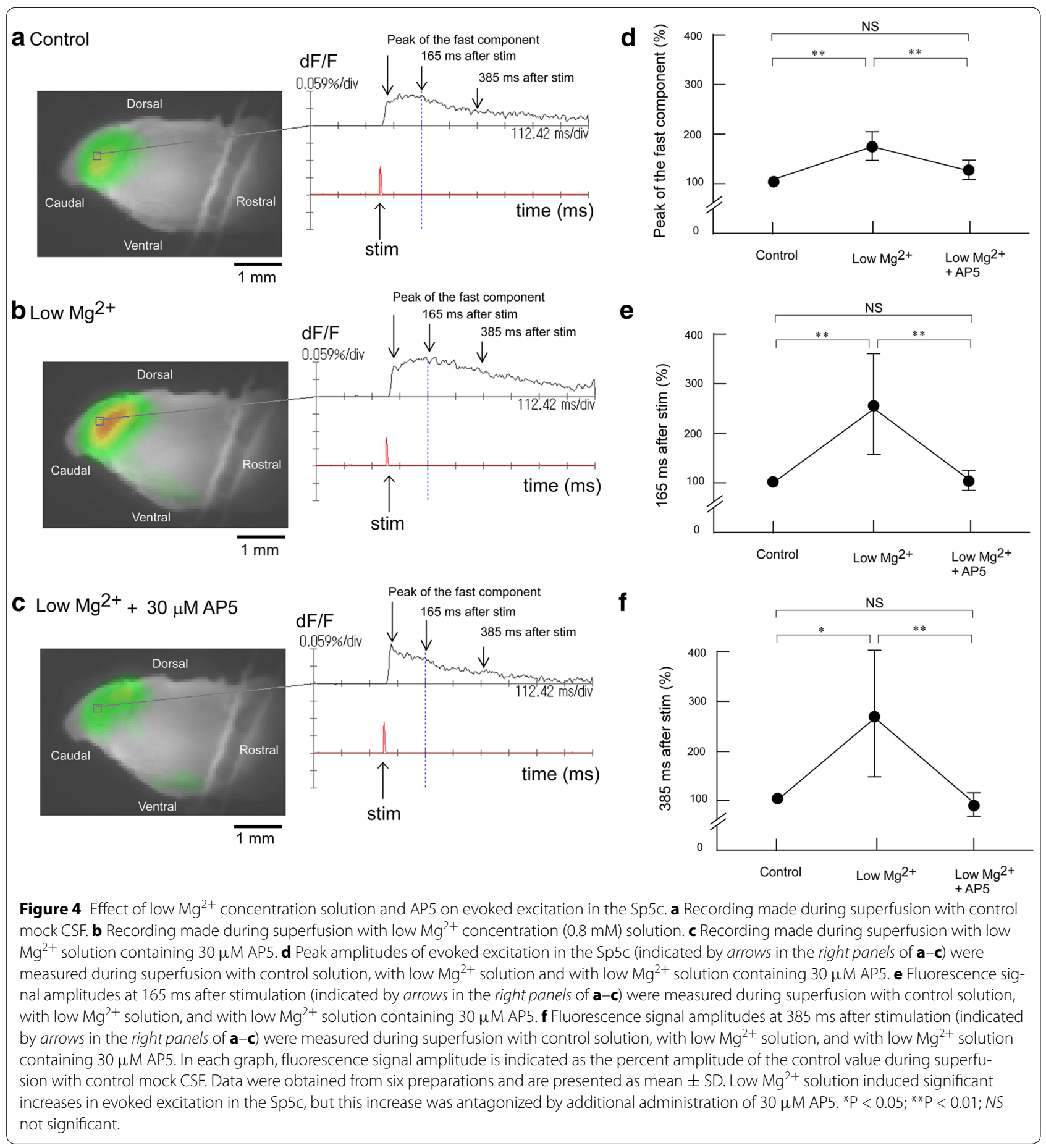

at $385 \mathrm{~ms}$ after stimulation) was more marked than that on the fast component $(128 \pm 23.0 \%)$. Additional administration of $100 \mu \mathrm{M}$ gabapentin in low $\mathrm{Mg}^{2+}$ conditions attenuated the evoked excitation to the level of the control conditions (Figure 6). The peak amplitude was $119 \pm 22.2 \%$ during superfusion (Figure $6 \mathrm{~d}$ ). The amplitude of the long-lasting component was also restored by additional superfusion with carbamazepine in low $\mathrm{Mg}^{2+}$ conditions. Amplitudes at 165 and $385 \mathrm{~ms}$ after stimulation decreased to $97.8 \pm 10.7 \%$ (Figure $6 \mathrm{e}$ ) and $115 \pm 12.4 \%$ (Figure $6 \mathrm{f}$ ), respectively. The effects of gabapentin in low $\mathrm{Mg}^{2+}$ conditions was similar to those of AP5 and carbamazepine. 


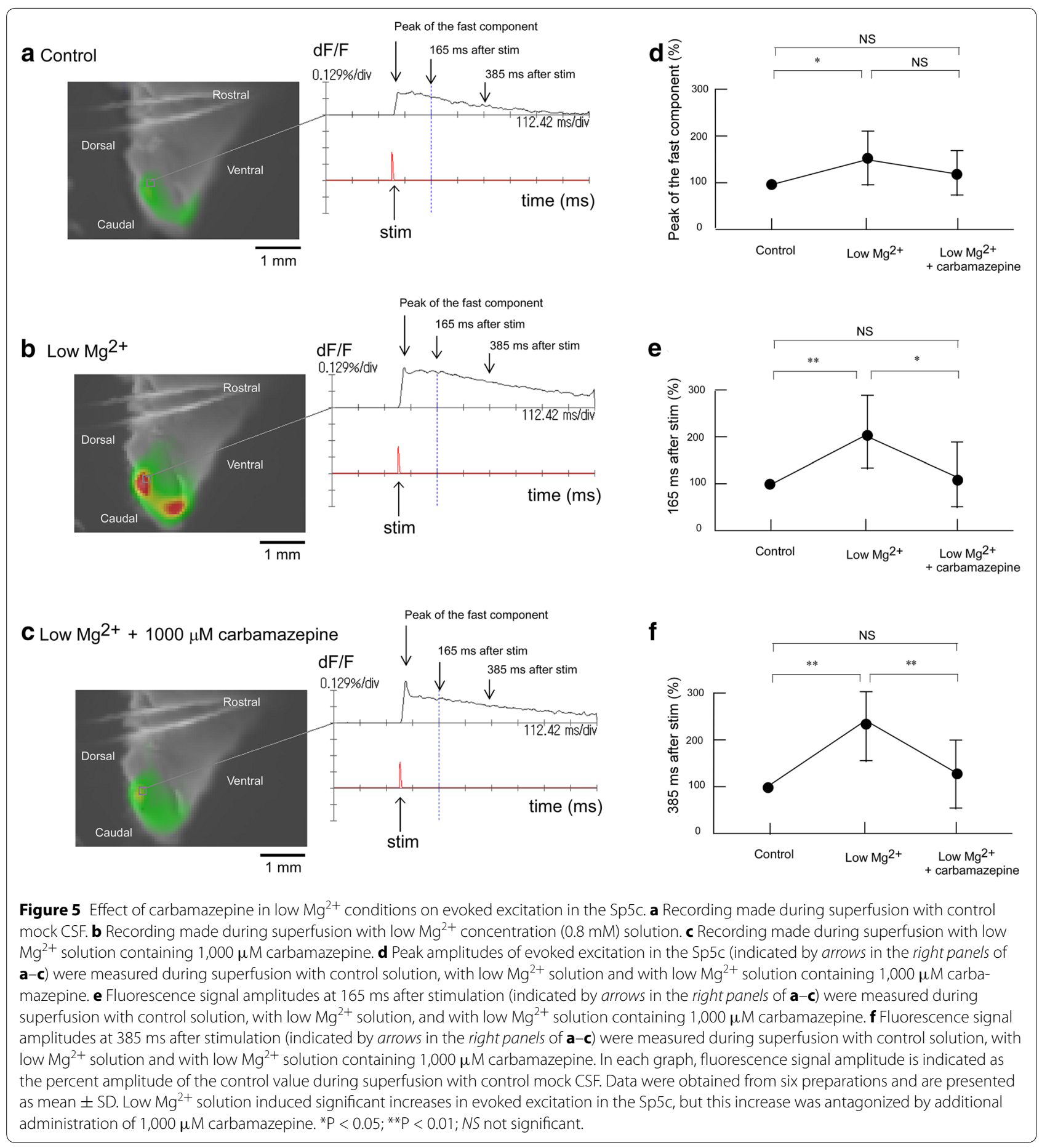

\section{Discussion}

Applying the voltage-sensitive dye imaging method with isolated neonatal rat brainstem slices allowed electrical trigeminal nerve stimulation-induced excitement of the Sp5c to be temporally and spatially visualized. Sole administration of carbamazepine and of low concentrations of gabapentin did not affect evoked excitation in the Sp5c, although administration of a high concentration of gabapentin emphasized the evoked excitation in the Sp5c. Superfusion with a low $\mathrm{Mg}^{2+}$ solution potentiated the evoked excitation. The effect of low $\mathrm{Mg}^{2+}$ conditions on the long-lasting component was more marked than that on the fast component. Additional administration of AP5, carbamazepine or gabapentin in low $\mathrm{Mg}^{2+}$ 


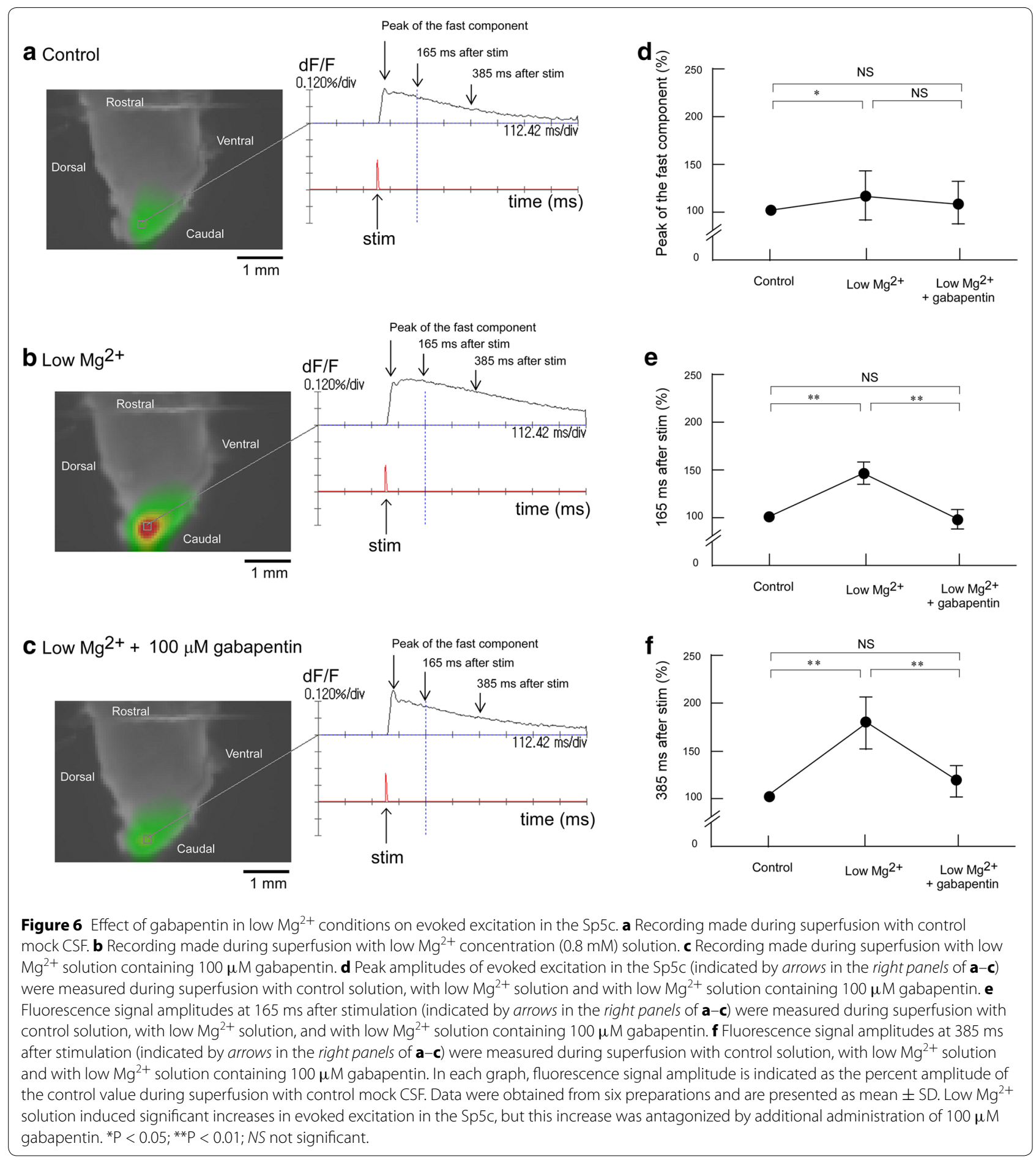

conditions attenuated the evoked excitation to the level of the control conditions, showing that the emphasized excitement by low $\mathrm{Mg}^{2+}$ concentration was antagonized by AP5, carbamazepine or gabapentin.

Single-pulse stimulation of the trigeminal nerve rootlet induced an optical response in the Sp5c. The optical signals were composed of two phases, a fast component with a sharp peak followed by a long-lasting component with a period of approximately $500 \mathrm{~ms}$. These findings were consistent with those of Takuma [19], who found that the spatiotemporal properties of optical signals were correlated with those of the field potential recordings by using similar 
preparations to ours. The optical signal of the fast component was eliminated by treatment with 6-cyano-nitro-quinoxaline-2,3-dione (CNQX). The long-lasting component increased in amplitude in low $\mathrm{Mg}^{2+}$ conditions but was significantly reduced by AP5. In an electrophysiological study using whole-cell patch-clamp techniques, Onodera et al. [16] reported that stimulation of the mandibular nerve at $0.03 \mathrm{~Hz}$ evoked compound excitatory postsynaptic potentials (EPSPs) of neurons in the Sp5c. Compound potentials consistent with monosynaptic EPSPs, which had a high threshold and with polysynaptic EPSPs, was attenuated by high frequency $(33-50 \mathrm{~Hz})$ stimulation. In low $\mathrm{Mg}^{2+}$ conditions, the fast monosynaptic EPSP component was abolished by simultaneous application of CNQX and AP5, and the slow polysynaptic EPSP was largely attenuated by application of AP5. In the present study, we confirmed the result that AP5 attenuated the fast component and largely attenuated the long-lasting component in low $\mathrm{Mg}^{2+}$ conditions. The result that enhanced excitation in low $\mathrm{Mg}^{2+}$ conditions was attenuated by application of AP5 suggested that low $\mathrm{Mg}^{2+}$ conditions induced the activation of NMDA receptors in the secondary neurons of the Sp5c. Furthermore, we demonstrated that carbamazepine and gabapentin had similar effects to AP5 on evoked excitation in the Sp5c in low $\mathrm{Mg}^{2+}$ conditions. This result strongly suggested that carbamazepine and gabapentin act as antagonists of NMDA receptors. Therefore, blockage of NMDA receptors of secondary neurons in the Sp5c may contribute to the clinical effectiveness of carbamazepine and gabapentin.

Inhibition of ion channels and synaptic transmission have been reported as the pharmacological actions of carbamazepine on the nervous system (see "Background"). In control conditions, sole application of carbamazepine did not affect evoked excitement in the Sp5c, suggesting that carbamazepine did not modulate the normal transduction of action potential and synaptic transmission. When excessive activation of the NMDA receptor is induced in the pathophysiology of several neurological conditions, such as trigeminal neuropathic pain, application of carbamazepine was effective because of a block in the activation of NMDA receptors [20,21].

Regarding the pharmacological action of gabapentin on the nervous system, the inhibition of active potential induction through binding to the $\alpha 2 \delta$ subunit of voltage-dependent $\mathrm{Ca}^{2+}$ channels has been reported [22]. Because gabapentin has a high affinity for the $\alpha 2 \delta$ subunit, it was considered to inhibit the release of neurotransmitters by inhibiting $\mathrm{Ca}^{2+}$ ion influx [11]. Gabapentin has also been shown to induce the modulation of other targets including NMDA receptors. In the present study, we suggested that the effective site of gabapentin was NMDA receptors in the Sp5c.
One unanticipated finding was that sole application of $100 \mu \mathrm{M}$ gabapentin emphasized the evoked excitation in control conditions. Petroff et al. [14] reported that gabapentin increased GABA levels in the brains of epileptic patients. Densely packed GABAergic neurons in the Sp5c have been described previously $[23,24]$. Because GABAergic transmission in various brain regions of immature animals is excitatory and inhibitory synaptic potentials appear relatively later in development [25], we should consider that not only non-GABAergic but also GABAergic interneurons in this region were excited. It may be possible that a high concentration of gabapentin induced GABA release, and GABA acts as an excitatory transmitter in the Sp5c. However, application of bicuculline further increased the evoked excitation after application of $100 \mu \mathrm{M}$ gabapentin (unpublished data), suggesting that GABAergic transmission was inhibitory in our preparations. Therefore, the possibility that GABAergic transmission in the $\mathrm{Sp} 5 \mathrm{c}$ is excitatory may be ruled out. To clarify the mechanisms of excitation by gabapentin, further studies are required.

In the present study, we estimated the conduction velocity as approximately $0.11 \mathrm{~m} / \mathrm{s}$ for the peak of the fast component. This value was similar to the value $(\sim 0.18 \mathrm{~m} / \mathrm{s})$ calculated by previous optical measurements [19]. These values were slow compared with a previous electrophysiological method, in which the conduction velocity was calculated to be $0.37 \mathrm{~m} / \mathrm{s}$ [16]. Conduction velocities obtained by optical and electrophysiological studies fall into the range of that of C-fibers. On the other hand, trigeminal sensory neurons changed electrophysiological properties during early postnatal maturation. Cabanes et al. [26] showed that trigeminal ganglion neurons in neonatal mice had uniformly slow conduction velocities and separated according to their conduction velocity into $\mathrm{A} \delta$ and $\mathrm{C}$ neurons during the 3 -week postnatal development period. Thus, it is difficult to show which axon type of a trigeminal nerve was mainly stimulated in the present study.

\section{Conclusion}

In conclusion, antiepileptic drugs carbamazepine and gabapentin did not decrease electrically evoked excitation in the $\mathrm{Sp} 5 \mathrm{c}$ in control conditions. Further excitation in low $\mathrm{Mg}^{2+}$ conditions was attenuated by the NMDA receptor antagonist AP5. Carbamazepine and gabapentin had similar effects to AP5 on evoked excitation in the Sp5c in low $\mathrm{Mg}^{2+}$ conditions. Thus, we concluded that carbamazepine and gabapentin act by blocking NMDA receptors in the Sp5c, which contributes to its antihypersensitivity action in neuropathic pain and trigeminal neuralgia. 


\section{Methods \\ Preparations}

All procedures were conducted in accordance with the guidelines of the Uekusa Gakuen University Laboratory Animal Care and Use Committee. Data were obtained from 54 neonatal Wistar rats (2-3 days old). The isolation of brainstem-spinal cord preparations has been described in detail previously [27]. In brief, rats were deeply anesthetized with diethyl ether and the brainstem was isolated in a dissecting chamber at room temperature. The chamber was filled with mock CSF equilibrated with a gas mixture $\left(5 \% \mathrm{CO}_{2}\right.$ in $\mathrm{O}_{2}$; $\mathrm{pH}$ 7.4). The composition of the mock CSF was as follows (in mM): $\mathrm{NaCl}, 126 ; \mathrm{KCl}, 5 ; \mathrm{CaCl}_{2}, 2 ; \mathrm{MgSO}_{4}, 2$; $\mathrm{NaH}_{2} \mathrm{PO}_{4}, 1.25 ; \mathrm{NaHCO}_{3}, 26$ and glucose, 30. The cerebrum was quickly removed by transection at the upper border of the inferior colliculus. Each trunk of the bilateral trigeminal nerves that run through the craniobasal bone was isolated to a length of $1 \mathrm{~mm}$, enabling it to be pulled into a suction electrode. Subsequently, the trigeminal nerve-attached brainstem-spinal cord was cut caudally at the level of the $\mathrm{C} 3$ roots (Figure $1 \mathrm{a}$ ). Furthermore, the isolated medulla was sectioned sagittally at $1-1.5 \mathrm{~mm}$ lateral from the midsagittal plane with a handmade slicer (Figure 1b). The trigeminal nerve-attached brainstem sagittal slice was placed in a recording chamber (volume $1.0 \mathrm{~mL}$ ) with the medial side up and continuously superfused (flow 4-6 mL/ min) at $26^{\circ} \mathrm{C}$ with oxygenated mock CSF.

\section{Voltage-sensitive dye imaging}

The voltage-sensitive dye imaging technique has been described in detail previously [28]. In brief, for staining, preparations were kept for $30 \mathrm{~min}$ in mock CSF containing the voltage-sensitive dye Di-4-ANEPPS $(7.5 \mathrm{mg} / \mathrm{mL}$ in $0.1 \%$ DMSO, Molecular Probes, Eugene, OR, USA), before being kept for at least $30 \mathrm{~min}$ in normal mock CSF. After staining, excess dye was removed by superfusion of the preparation with dye-free solution. After $30 \mathrm{~min}$ of washing, optical imaging and data analysis were performed using a MiCAM02 hardware and software package (BrainVision, Tokyo, Japan). For optical imaging, we used a fixed-stage upright fluorescence microscope (Measurescope UM-2, Nikon, Tokyo, Japan) with a low magnification objective lens (XL Fluor $4 \times 1340$, Olympus, Tokyo, Japan) and a high-resolution MiCAM02 camera.

To record the voltage-sensitive dye signals, we used light from a $150 \mathrm{~W}$ halogen lamp controlled by an electromagnetic shutter (Oriel Instruments, Stratford, USA). Changes in fluorescence of the dye were detected by the camera through a 510-560 nm excitation filter, a dichroic mirror, and a $590 \mathrm{~nm}$ absorption filter (MBE1405, Nikon). The camera captured images of $88 \times 60$ pixels, and the size of the area was $5.4 \times 3.7 \mathrm{~mm}$. Optical signals from $3 \times 3$ pixels (approximately $0.03 \mathrm{~mm}^{2}$ ) were averaged and are showed in the images.

Total frame acquisition was set to 511. Sampling time was $2.2 \mathrm{~ms} /$ frame; therefore, the total recording time was $1,124.2 \mathrm{~ms}$. Neuronal activity was evoked by square pulse electrical stimuli (1.0 ms, $0.5-1.0 \mathrm{~mA})$ delivered to the trigeminal nerve rootlet via a glass suction electrode. Acquisition was triggered by the electrical stimulus. The trigger signal was activated after one-quarter of the total recording time, corresponding to $284 \mathrm{~ms}$ after starting acquisition when we set the total acquisition time to 1,124.2 ms. Signal amplitude was normalized using the $\mathrm{d} F / F$ method, where $F$ is the total fluorescent signal and $\mathrm{d} F$ corresponds to the change in fluorescence observed following evoked modification of the membrane potential. To improve the signal-to-noise ratio, we averaged signals detected in 10 consecutive trials at $0.3 \mathrm{~Hz}$. To analyze the intensity of the signals, we measured the peak amplitude (at 30-40 ms after stimulation) and amplitudes at $40 \%$ (at $165 \mathrm{~ms}$ after stimulation) and at $60 \%$ (at $385 \mathrm{~ms}$ after stimulation) of the total recording time (1,124.2 ms). Measuring points at 165 and $385 \mathrm{~ms}$ after stimulation were selected arbitrarily.

\section{Drug administration}

Carbamazepine (Sigma Aldrich, Saint Louis, MO, USA) was added to perfused mock CSF at 10, 100 and $1,000 \mu \mathrm{M}$. Gabapentin (Sigma Aldrich) was added to perfused mock CSF at 1.0, 10 and $100 \mu \mathrm{M}$. NMDA receptor antagonist DL-2-amino-5-phosphonopentanoic acid (AP5, Sigma Aldrich) was added to perfused mock CSF at $30 \mu \mathrm{M}$. These concentrations were determined from preliminary experiments and previous studies [16, 22]. Optical records using electrical stimulation were taken $20 \mathrm{~min}$ after the start of superfusion with control mock CSF and were taken $20 \mathrm{~min}$ after switching to drug-containing mock CSF. To induce activation of the NMDA receptor, we used low $\mathrm{Mg}^{2+}$ concentration solution (in $\mathrm{mM}$ ): $\mathrm{NaCl}, 126 ; \mathrm{KCl}, 5 ; \mathrm{CaCl}_{2}, 2.6 ; \mathrm{MgSO}_{4}, 0.8 ; \mathrm{NaH}_{2} \mathrm{PO}_{4}, 1.25$; $\mathrm{NaHCO}_{3}, 26$ and glucose, 30. In these series of experiments, optical records using electrical stimulation were taken 20 min after the start of superfusion with control mock CSF and were taken 20 min after switching to low $\mathrm{Mg}^{2+}$ concentration solution, and then, taken $20 \mathrm{~min}$ after switching to low $\mathrm{Mg}^{2+}$ solution containing $30 \mu \mathrm{M}$ AP5, 1,000 $\mu \mathrm{M}$ carbamazepine or $100 \mu \mathrm{M}$ gabapentin.

\section{Data analysis}

Optical signal amplitudes obtained before superfusion with mock CSF containing drugs was defined as the control value. The level of statistical significance for the difference between the mean value of each variable 
obtained during application of different concentrations of drug was conducted by ANOVA followed by pair-wise comparisons using the Tukey-Kramer method for multiple comparisons as indicated. All statistical analyses were conducted using Statcel (OMS publisher, Japan). All values were reported as mean $\pm \mathrm{SD}$, and all $P$ values $<0.05$ were considered significant.

\section{Authors' contributions}

AM and HA carried out experiments, collected and analyzed data, and drafted the manuscript. AM and HA contributed equally to this study. YH carried out experiments and conducted the study. SS and KY helped design and conduct the study, and drafted the manuscript. TS and NU carried out experiments and collected and analyzed data. SK designed and supervised this study, explained the data, and drafted the manuscript. All authors read and approved the final manuscript.

\section{Author details}

${ }^{1}$ Division of Anesthesiology, Department of Clinical Care Medicine, Kanagawa Dental College, Yokosuka, Kanagawa 238-8580, Japan. ${ }^{2}$ Department of Anesthesiology, Kitasato University School of Medicine, Sagamihara, Kanagawa 252-0375, Japan. ${ }^{3}$ Center for Medical Sciences, Ibaraki Prefectural University of Health Sciences, Ami, Inashiki-gun, Ibaraki 300-0394, Japan. ${ }^{4}$ Faculty of Health Sciences, Uekusa Gakuen University, Ogura-cho, Wakaba-ku, Chiba 264-0007, Japan.

\section{Acknowledgements}

This work was supported by JSPS KAKENHI Grant Numbers 23590719, 26460704, by the Science Research Promotion Fund of the Promotion and Mutual Aid Corporation for Private Schools of Japan, and by the Science Research Fund of the Uekusa Gakuen University.

\section{Compliance with ethical guidelines}

\section{Competing interests}

The authors declared that they have no competing interests.

Received: 19 September 2014 Accepted: 6 July 2015

Published online: 21 July 2015

\section{References}

1. Lemos L, Alegria C, Oliveira J, Machado A, Oliveira P, Almeida A. Pharmacological versus decompression approaches for treatment of trigeminal neuralgia: clinical outcomes and direct costs. J Pain Res. 2011;4:233-44.

2. Lemos L, Fontes R, Frores S, Oliveira P, Almeida A. Effectiveness of the association between carbamazepine and peripheral analgesic block with ropivacaine tor the treatment of trigeminal neuralgia. J Pain Res. 2010;3:201-12.

3. Worley PF, Baraban JM. Site of anticonvulsant action on sodium channels: autoradiographic and electrophysiological studies in rat brain. Proc Natl Acad Sci USA. 1987;84:3051-5.

4. Ambrósio AF, Soares-Da-Silva P, Carvalho CM, Carvalho AP. Mechanisms of action of carbamazepine and its derivatives, oxcarbazepine, BIA 2-093, and BIA 2-024. Neurochem Res. 2002;27:121-30.

5. Uemura Y, Fujita T, Ohtsubo S, Hirakawa N, Sakaguchi Y, Kumamoto E. Effects of various antiepileptics used to alleviate neuropathic pain on compound action potential in frog sciatic nerves: comparison with those of local anesthetics. Biomed Res Int. 2014;2014 (Art. ID 540238). doi:10.1155/2014/540238

6. Todorovic SM, Lingle CJ. Pharmacological properties of T-type $\mathrm{Ca}^{2+}$ current in adult rat sensory neurons: effects of anticonvulsant and anesthetic agents. Neurophysiol. 1998;79:240-52.
7. Zona C, Tancredi V, Palma E, Pirrone GC, Avoli M. Potassium currents in rat cortical neurons in culture are enhanced by the antiepileptic drug carbamazepine. Can J Physiol Pharmacol. 1990;68:545-7.

8. Okada M, Kawata Y, Mizuno K, Wada K, Kondo T, Kaneko S. Interaction between $\mathrm{Ca}^{2+}, \mathrm{K}^{+}$, carbamazepine and zonisamide on hippocampal extracellular glutamate monitored with a microdialysis electrode. $\mathrm{Br} J$ Pharmacol. 1998;124:1277-85.

9. Biber K, Fiebich BL, Gebicke-Härter P, van Calker D. Carbamazepineinduced upregulation of adenosine A1-receptors in astrocyte cultures affects coupling to the phosphoinositol signaling pathway. Neuropsychopharmacology. 1999;20:271-8.

10. Cheng JK, Chiou LC. Mechanisms of the antinociceptive action of gabapentin. J Pharmacol Sci. 2006;100:471-86.

11. Kukkar A, Bali A, Singh N, Jaggi AS. Implications and mechanism of action of gabapentin in neuropathic pain. Arch Pharm Res. 2013;36:237-51.

12. Sutton KG, Martin DJ, Pinnock RD, Lee K, Scott RH. Gabapentin inhibits high-threshold calcium channel currents in cultured rat dorsal root ganglion neurons. Br J Pharmacol. 2002;135:257-65.

13. Kim YS, Chang HK, Lee JW, Sung YH, Kim SE, Shin MS, et al. Protective effect of gabapentin on $\mathrm{N}$-methyl-D-aspartate-induced excitotoxicity in rat hippocampal CA1 neurons. J Pharmacol Sci. 2009;109:144-7.

14. Petroff OA, Hyder F, Rothman DL, Mattson RH. Effects of gabapentin on brain GABA, homocarnosine, and pyrrolidinone in epilepsy patients. Epilepsia. 2000;41:675-80.

15. Hamba M, Onodera K, Takahashi T. Long-term potentiation of primary afferent neurotransmission at trigeminal synapses of juvenile rats. Eur J Neurosci. 2000;12:1128-34.

16. Onodera K, Hamba M, Takahashi T. Primary afferent synaptic responses recorded from trigeminal caudal neurons in a mandibular nerve-brainstem preparation of neonatal rats. J Physiol. 2000;524:503-12.

17. Seo K, Fujiwara N, Takeuchi K, Maeda T, Someya G. Optical imaging of excitation propagation evoked by stimulation to the trigeminal caudalis. NeuroReport. 2001;12:3985-8.

18. Seo K, Fujiwara N, Takeuchi K, Maeda T, Someya G. Repetitive afferent stimulation propagates excitation in the trigeminal caudalis. NeuroReport. 2003;14:1321-5.

19. Takuma S. Effect of neonatal capsaicin treatment on neural activity in the medullary dorsal horn of neonatal rats evoked by electrical stimulation to the trigeminal afferents: an optical, electrophysiological, and quantitative study. Brain Res. 2001;906:1-12.

20. Landmark CJ. Targets for antiepileptic drugs in the synapse. Med Sci Monit. 2007;13:RA1-7

21. Hahm TS, Ahn HJ, Ryu S, Gwak MS, Choi SJ, Kim JK, et al. Combined carbamazepine and pregabalin therapy in a rat model of neuropathic pain. $\mathrm{Br} J$ Anaesth. 2012;109:968-74

22. D'Mello R, Dickenson AH. Spinal cord mechanisms of pain. $\mathrm{Br} J$ Anaesth. 2008;101:8-16.

23. Kuwana S, Tsunekawa N, Yanagawa Y, Okada Y, Kuribayashi J, Obata K. Electrophysiological and morphological characteristics of GABAergic respiratory neurons in the mouse pre-Bötzinger complex. Eur J Neurosci. 2006;23:667-74.

24. Viggiano A, Monda M, Viggiano A, Chiefari M, Aurilio C, De Luca B. Evidence that GABAergic neurons in the spinal trigeminal nucleus are involved in the transmission of inflammatory pain in the rat: a microdialysis and pharmacological study. Eur J Pharmacol. 2004;496:87-92.

25. Ben-Ari Y. The GABA excitatory/inhibitory developmental sequence. Neuroscience. 2014;279:187-219.

26. Cabanes C, de López Armentia M, Viana F, Belmonte C. Postnatal changes in membrane properties of mice trigeminal ganglion neurons. J Neurophysiol. 2002;87:2398-407.

27. Kuwana S, Okada Y, Natsui T. Effects of extracellular calcium and magnesium on central respiratory control in the brainstem-spinal cord of neonatal rat. Brain Res. 1998;786:194-204.

28. Okada Y, Chen Z, Yoshida H, Kuwana S, Jiang W, Maruiwa H. Optical recording of the neuronal activity in the brainstem-spinal cord: application of a voltage-sensitive dye. Adv Exp Med Biol. 2001;499:113-8. 